\title{
Biocatalytic and Biological Activities of Cassia Occidentalis Mediated Silver Nanoparticles
}

\author{
Shashika Singh and Viresh Mohanlall* \\ Department of Biotechnology and Food Technology, Faculty of Applied Sciences, \\ Durban University of Technology, Steve Biko Campus, Durban 4001, South Africa
}

('Corresponding author's e-mail: vireshm@dut.ac.za)

Received: 14 August 2020, Revised: 18 June 2021, Accepted: 14 June 2021

\begin{abstract}
Silver nanoparticles (AgNPs) were synthesized using the leaf extracts from Cassia occidentalis. The reactions were marked by a distinct colour change and the formation of AgNPs was monitored by measuring the UV-Vis spectra. The morphology and crystalline phase of the metal nanoparticles were determined using Transmission Electron Microscopy (TEM) and Scanning Electron Microscopy (SEM) with Energy Dispersive X-ray analysis (EDX) and Fourier-Transform-Infrared Spectroscopy (FTIR). The biocatalytic activity of the AgNPs against nitrifying compounds was determined using 4-nitrophenol and 4-nitroalanine. The biological screening involved analyzing the AgNPs effectiveness against gramnegative and gram-positive bacteria (Escherichia coli, Staphylococcus aureus, Klebsiella pneumoniae, Pseudomonas aeruginosa, Bacillus cereus and Enterococcus faecalis). The AgNPs displayed minimal inhibition at low concentrations ranging from 25 to $50 \mu \mathrm{g} / \mathrm{mL}$. The toxicity of the AgNPs was tested using the brine shrimp assay and was observed as non-toxic with a nearly zero mortality rate. The AgNPs exhibited an antioxidant potential of $70.90 \%$ inhibition at $1000 \mu \mathrm{g} / \mathrm{mL}$. C. occidentalis is noted as a potential bioresource for synthesizing AgNPs with applications as antibacterial, antioxidant and biocatalytic agents.
\end{abstract}

Keywords: Biocatalytic agents, Cassia occidentalis, Antibacterial, Antioxidant, Silver nanoparticles

\section{Introduction}

Nanotechnology is the concept of manipulating matter at an atomic level, according to initial research by Nobel Laureate Richard Feynman in 1959, at the California Institute of Technology [1], where he gave a lecture entitled "There's Plenty of Room at the Bottom: An Invitation to Enter a New Field of Physics". There is a technological revolution on the proverbial horizon and, although being a relatively new field in science, nanotechnology is the driving force behind it. The beginning of the $21^{\text {st }}$ century saw a rapid increase in interest in the emerging fields of nanoscience and nanotechnology. The application of nanotechnology is diverse, playing a role in the pharmaceutical, agricultural [2] and medical field as well as in optical receptors, biosensors and biomaterial production, to name a few [3]. Nanoparticles are comprised of a multitude of materials. This includes carbon base nanoparticles, ceramic nanoparticles, semiconductor nanoparticles, polymeric nanoparticles and metal nanoparticles. These materials combined are responsible for the unique properties of the nanoparticles formed. These properties can each be used to advance the field of science immensely. This includes the fields of drug delivery systems and cancer treatments as well as treatments for glaucoma.

Currently, there is much interest surrounding the implications of this technology, in its beneficial qualities as well as its drawbacks. The initial chemical synthesis of nanoparticles requires the use of highly toxic chemicals and results in harmful by-products [3,4]. The synthesis of metal nanoparticles (MNPs) and their potentially hazardous consequences have been a cause for concern and the search for safer and cheaper methods are continuously being explored. The synthesis of MNPs with the aid of plant materials removes the use of harmful toxic chemicals and decreases its harmful effects on the environment.

The use of $C$. occidentalis in the synthesis of MNPs may prove beneficial in decreasing the use of harmful chemicals by utilizing the plant's natural chemical components. C. occidentalis leaves have been known to display anticarcinogenic and antibacterial activity and contain a chemical composition 
of sennoside, polysaccharides, fatty oils, and flavonoid glycosides, among other metabolites [4]. C. occidentalis has been used in several studies to determine its applications and properties [5-8].

AgNPs exhibit a wide range of biological activity due to their shape, size, surface chemistry, coating or capping agents, their efficiency of ion release and the reducing agents used in the synthesis process [9]. AgNPs were found to exhibit antimicrobial properties and are utilised in many pharmaceutical products to treat wounds and prevent bacterial infection [4]. The synthesis of AgNPs was achieved using $C$. occidentalis. Thereafter the AgNPs were characterized using Transmission Electron Microscopy, Scanning Electron Microscopy with EDX, UV-Vis spectrophotometry and IR spectroscopy. The biocatalytic and biological activities were tested to determine the efficacy of green synthesis in creating efficient and effective AgNPs.

\section{Materials and methods}

\section{Materials}

Mueller Hinton Agar plates, Microtiter plates and Mueller Hinton Broth (MHB) were purchased from Bio-Rad Laboratories (Richmond, VA and USA). Bacterial cultures were obtained from Lancet Laboratories (SA). Microbank vials were purchased from Davies diagnostic, (SA). All chemicals were of analytical grade and were supplied by Sigma-Aldrich (St Louis, MO and USA).

\section{Collection of plant and preparation of leaf extracts}

C. occidentalis was obtained from around Durban, Kwa-Zulu Natal and South Africa. The leaves were rinsed 3 times with distilled water to remove all foreign particles. The leaf solution was thereafter prepared by cutting $60 \mathrm{~g}$ of leaves and adding $300 \mathrm{~mL}$ of deionized water. The deionized water and leaf mixture was then placed in a water bath at $60^{\circ} \mathrm{C}$ shaking at $30 \mathrm{rpm}$ for $1 \mathrm{~h}$. The extract was cooled down and filtered with Whatman No. 1 filter paper. The leaf extract was stored in a refrigerator at $4{ }^{\circ} \mathrm{C}$. The leaf extract was used within a week of preparation [4] and a new extract was made when it was necessary.

\section{Green synthesis of silver nanoparticles}

The silver nanoparticles (AgNPs) were synthesized by adding the plant extract from $C$. occidentalis to prepared $4 \mathrm{mM}$ silver nitrate, $1 \mathrm{~mL}$ of plant extract was added to $24 \mathrm{~mL}$ of silver nitrate. This solution was placed in an $80{ }^{\circ} \mathrm{C}$ - water bath for $6 \mathrm{~h}$. The reaction was monitored using the UV-vis spec at hourly intervals. The optimal time for subsequent synthesis was determined to be $4 \mathrm{~h}$. The bottle was covered with foil to minimize any exposure to light. The end of the reaction was noted with a distinct colour change to a darker shade of brown [4]. The method of obtaining the plant extract and synthesizing the nanoparticles was loosely based on the methods used by Gondwal and Pant (2018), minor changes were made to establish a more efficient green method of synthesis, where previously it took $24 \mathrm{~h}$ to synthesize AgNPs, with this modified method it takes $4 \mathrm{~h}$.

\section{Characterization of the synthesized nanoparticles}

Ultra-violet (UV) spectroscopy

The formation and stability of AgNPs were analyzed by UV-Visible spectroscopy using a Varian Cary $100 \mathrm{UV}$-Vis spectrophotometer containing Win UV software. A scan in the wavelength range of 200 to $800 \mathrm{~nm}$ was carried out. Samples were poured into quartz cuvettes, placed into the spectrophotometer and scanned in the aforementioned wavelength range. Thereafter, the absorbance measurement in relative light units (AU) were recorded.

\section{Transmission electron microscopy (TEM)}

TEM was used to determine the morphology and particle size of the synthesized AgNPs. Sample preparation involved centrifugation of the AgNP solution at $1300 \mathrm{rpm}$ for $45 \mathrm{~min}$, followed by discarding of the supernatant, and re-suspending of the precipitant in distilled water. From this, a drop of each sample was then placed on a carbon-coated copper grid and allowed to air dry at room temperature before loading into the Transmission Electron Microscope for analysis. The microscope used was a JEOL JEM1010 Transmission Electron Microscope with a Megaview III camera and iTEM UIP software (Tokyo and Japan) accessed from the University of Kwa-Zulu Natal (Westville Campus and South Africa). 


\section{Scanning electron microscopy (SEM) and energy dispersive $x$-ray analysis (EDX)}

To investigate the presence of silver nanoparticles and for observing the particle image, a drop of the silver nanoparticles was placed on an aluminium stub, and then allowed to air dry, this permits the water to evaporate and be viewed using a ZEISS LEO 1450 Scanning Electron Microscope (conventional SEM and X-ray microanalysis) at the University of Kwa-Zulu Natal. For elemental analysis, the nanoparticles were subjected to energy dispersive X-Ray analysis coupled with SEM.

\section{Fourier-transform infrared (FTIR) spectroscopy}

FTIR analyses were used to identify if the same biomolecules present in the plant extract were responsible for the synthesis and capping of the AgNPs. At the onset, sample preparations involved centrifugation of AgNP solutions at $1300 \mathrm{rpm}$ for $45 \mathrm{~min}$, followed by discarding of the supernatant, and re-suspending of the precipitants in distilled water. From this, a drop of the sample was then placed on a Perkin Elmer, Spectrum 100 FTIR spectrophotometer and a scan measuring transmittance percentage $(\% \mathrm{~T})$ over the range of wavenumbers from 500 to $4000 \mathrm{~cm}^{-1}$ was taken. Thereafter, E-FTIR software was used to analyze the results and ascertain peak values which were then interpreted.

\section{Biological and biocatalytic activities of synthesized nanoparticles \\ Catalytic reduction of 4-nitrophenol and 4-nitroaniline}

The synthesized AgNPs were used as a catalyst for the reduction of nitro organic compounds, 4nitrophenol and 4-nitroaniline to amino compounds by sodium borohydride [32]. Into a $3 \mathrm{~mL}$ cuvette, containing freshly prepared sodium borohydride $(1 \mathrm{~mL}, 0.2 \mathrm{M})$ solution, 4-nitrophenol (1.9 $\mathrm{mL}, 0.2 \mathrm{mM})$ solution was added. The cuvette was then placed in a UV-Vis spectrophotometer and the absorbance was recorded between a wavelength range of 200 to $800 \mathrm{~nm}$. After adding metal nanoparticles $(0.1 \mathrm{~mL}, 0.1 \%)$ solution, the cuvette was shaken vigorously for mixing and kept in a UV-Vis spectrophotometer and scanned from the above-mentioned wavelength range.

\section{Antibacterial activity of the AgNPs}

Synthesized silver nanoparticles were tested for antimicrobial activity by disc diffusion method against pathogenic bacteria, Klebsiella pneumonia (ATCC 70603), Pseudomonas aeruginosa (ATCC 27853), Bacillus cereus (ATCC 10876), Enterococcus faecalis (ATCC 29212), Staphylococcus aureus (ATCC 25923) and Escherichia coli (ATCC 25922). The sterilized discs were dipped in different concentrations of silver nanoparticle dispersion in dimethyl sulfoxide (DMSO), namely, $100 \%$ (1000 $\mu \mathrm{g} / \mathrm{mL}), 50 \%(500 \mu \mathrm{g} / \mathrm{mL})$, and $25 \%(250 \mu \mathrm{g} / \mathrm{mL})$, and dried in an oven at $30-40{ }^{\circ} \mathrm{C}$. The silver nanoparticle impregnated discs were placed on the plates and kept for incubation at $37^{\circ} \mathrm{C}$ for $24 \mathrm{~h}$. After incubation, the different levels of zones of inhibition of bacteria were measured. The standard antibiotic drug, ciprofloxacin was used as a positive control and $10 \%$ DMSO was used as a negative control. The experiments were done in triplicate and mean values of zone diameter were taken.

\section{Cytotoxicity assay-brine shrimp assay}

Artificial seawater was made by dissolving $23 \mathrm{~g}$ of NaCl, $11 \mathrm{~g} \mathrm{MgCl}_{2} \cdot 6 \mathrm{H}_{2} \mathrm{O}, 4 \mathrm{~g} \mathrm{Na}_{2} \mathrm{SO}_{4}, 1.3 \mathrm{~g}$ $\mathrm{CaCl}_{2} \cdot 2 \mathrm{H}_{2} \mathrm{O}$ and $0.7 \mathrm{~g} \mathrm{KCl}$ in $1000 \mathrm{~mL}$ of distilled water. The $\mathrm{pH}$ was adjusted to 9 using $\mathrm{Na}_{2} \mathrm{CO}_{3}$ and the mixture was autoclaved. A yeast extract was also prepared as a source of food for the brine shrimp. The autoclaved seawater was added to a sterilized glass container and the brine shrimp eggs were added thereafter. After $24 \mathrm{~h}$ the eggs began to hatch, and 3 drops of yeast extract were added as a source of nutrition. After $48 \mathrm{~h}$, the hatched brine shrimp were removed from the tank and placed into wells, 10 brine shrimp per well. The AgNPs was redispersed using ethanol and were further diluted. Each well containing 10 brine shrimp was then inoculated with $100 \mu \mathrm{L}$ of each dilution of nanoparticle as well as a plant extract and silver solutions individually. The negative and positive control was ethanol and potassium chromate, respectively. The wells were observed at hourly intervals for $4 \mathrm{~h}$ and thereafter at the $20 \mathrm{~h}$ mark and finally at the $24 \mathrm{~h}$ mark. All tests were done in triplicate.

\section{Antioxidant assay}

The AgNPs were screened for free radical scavenging activity by the DPPH method [11]. The AgNPs dispersed in methanol $(10-100 \mu \mathrm{g} / \mathrm{mL})$ were added to different test tubes and the volume was made up to $4 \mathrm{~mL}$ using methanol, separately. Then, $3 \mathrm{~mL}$ of DPPH $(0.1 \mathrm{mM})$ solution was added and incubated in a dark room for about $30 \mathrm{~min}$ at room temperature. All the solutions were prepared in the laminar flow. The scavenging activity on the DPPH radical was determined by measuring the absorbance 
at $517 \mathrm{~nm}$ against a blank with a UV-Vis spectrophotometer. Gallic acid was used as the positive control. Tests were performed in triplicate and the results were averaged.

$\%$ of radical scavenging activity $=\left[A_{\text {control }}-\frac{A_{\text {sample }}}{A_{\text {control }}}\right] \times 100$

where $A_{\text {control }}$ is the absorbance of the control sample (DPPH solution without sample) and $A_{\text {sample }}$ is the absorbance of the sample (DPPH solution and sample).

\section{Statistical analysis}

Antibacterial activity studies were performed in triplicate and the results expressed as mean \pm standard deviation (S.D). Data analyzed by 2-way ANOVA and t-test using GraphPad Prism 6.0 and statistically significant values are indicated by $\mathrm{p}<0.0$.

\section{Results and discussion}

\section{Synthesis of AgNPs, UV-Vis spectroscopy SEM and TEM analysis}

Metal nanoparticles are well known for their unique properties, particularly, optical properties, as a result of surface plasmon resonance (SPR). Thus, the successful formation of AgNPs was confirmed with colour change and UV-Vis spectroscopy. The AgNPs were prepared by mixing plant extract of $C$. occidentalis with $\mathrm{AgNO}_{3}$ solution which resulted in a successful synthesis. This was indicated by the colour change of the reaction mixture from colourless solution to yellowish-brown solution. The colour change can be attributed to the reduction of silver ions $\left(\mathrm{Ag}^{+}\right)$from silver nitrate into AgNPs by active biomolecules present in the plant extract [12]. The colour of AgNPs is due to the excitation of SPR arising due to the collective oscillation of free conduction electrons induced by an interacting electromagnetic field [13]. Furthermore, the characteristic absorption band of AgNPs is known to range between 400 to $460 \mathrm{~nm}$ [14]. The absorption bands observed for tested sample preparations ranged between 420 and $470 \mathrm{~nm}$ (Figure 1), thus confirming the successful preparation of AgNPs by this extract.

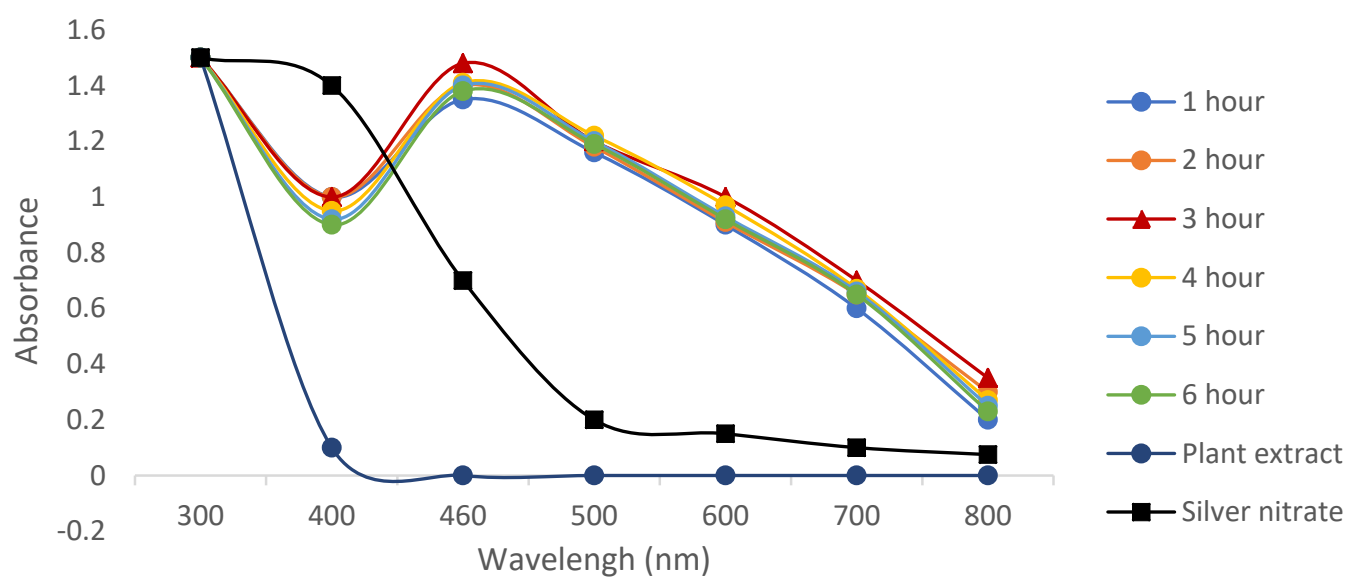

Figure 1 UV-Vis absorption spectra of synthesized silver nanoparticles (AgNPs).

SEM micrographs of the synthesized AgNPs of $C$. occidentalis magnified at $\times 50,000, \times 100,000$ and measured at 100 and $200 \mathrm{~nm}$ are shown in Figure 2. The triangular, pentagonal and hexagonal structures are present. Elemental composition analysis using Energy Dispersive X-Ray Analysis (EDX) proves the chemical purity of the synthesized AgNPs (Figure 3(a)). The elemental analysis of the AgNPs shown in the figure revealed a strong silver signal $(70.78 \%)$ along with weak signals of $\mathrm{O}(5.42 \%), \mathrm{Cl}(8.52 \%)$ and $\mathrm{C}(14.89 \%)$. Furthermore, one small peak of $\mathrm{Al}(0.38 \%)$ was also observed in the green synthesized silver nanoparticle sample. The presence of $\mathrm{C}, \mathrm{O}$ and $\mathrm{Cl}$ may be denoted to components from the plant 
extract that was used to synthesize the AgNPs. The Al is visible in trace amounts, due to the sample preparation which involves air-drying the sample on an aluminium surface, due to the removal process from the surface, trace elements of $\mathrm{Al}$ were present in the analysis. A large presence of $\mathrm{C}$ is also explained due to the sample analysis taking place on a carbon grid [33]. The electron microscopic study of the nanoparticles using TEM revealed that the AgNPs predominates with spherical, triangular nanoprism, decahedral and quasi-spherical morphologies ranging from 10 to $90 \mathrm{~nm}$ with an average size of 35$50 \mathrm{~nm}$ (Figure 4(a)-4(c)). These measurements were obtained during the TEM analysis by directly comparing the size of a few particles to the scale provided and determining an average. The SEM results agree as compared to research conducted by Gondwal and Pant (2018) who reported that the SEM micrographs clearly show aggregates of AgNPs of $C$. occidentalis with average particle sizes ranging from 20 to $65 \mathrm{~nm}$. The AgNPs efficiency may be affected due to agglomeration of the nanoparticles since it reduces the surface free energy as a result of increasing their size. This increase in size affects the toxicity of the AgNPs. The AgNP is required to be extremely small and singular to sufficiently disrupt a cell and the agglomeration of the particles may make it difficult to enter the cell, thus decreasing the toxicity [34].
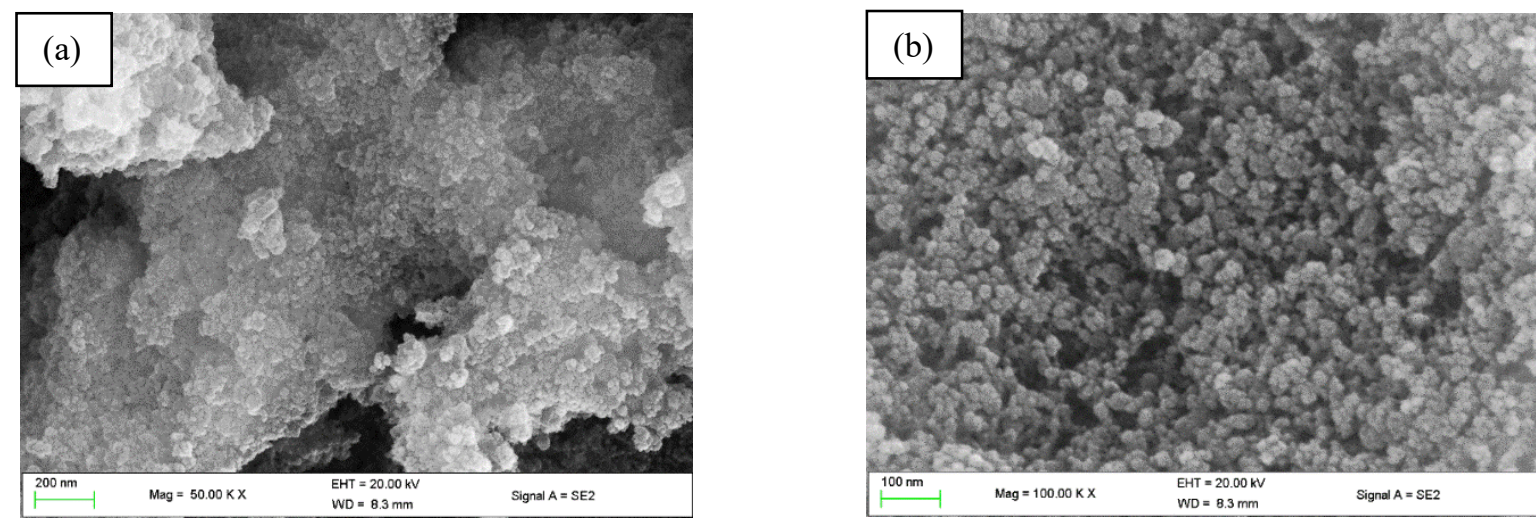

Figure 2 SEM micrograph of synthesized AgNPs at $50 \mathrm{kX}$ (a) and $100 \mathrm{kX}$ magnification (b).
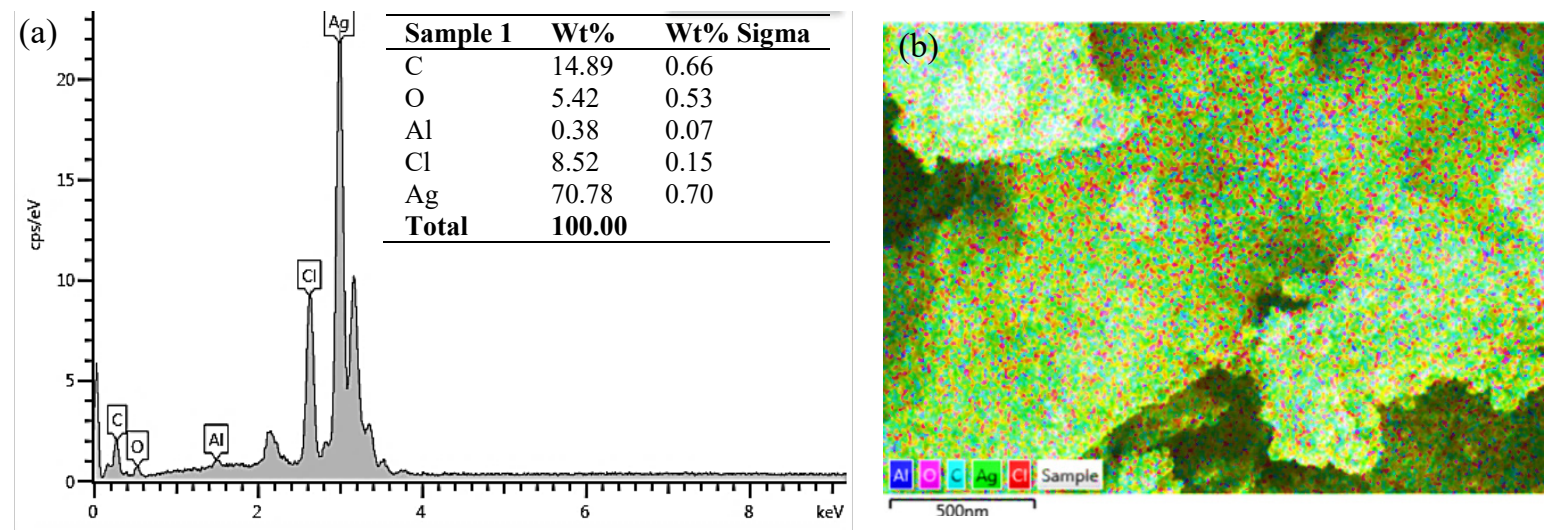

Figure 3 (a) - elemental composition of AgNPs using Scanning Electron Microscopy (SEM) and Energy Dispersive X-Ray Analysis (EDX), (b) - elemental map showing the presence of aluminium, oxygen, carbon, silver and chlorine. 

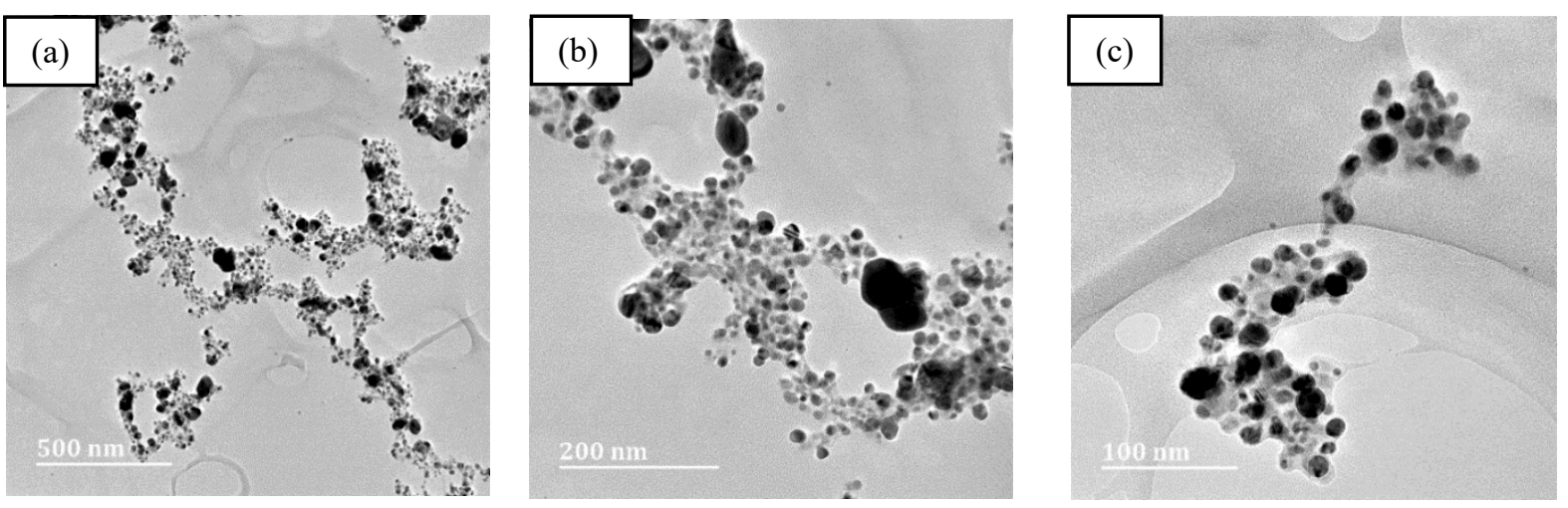

Figure 4 TEM micrograph of AgNPs using C. occidentalis at the scale bar corresponding to (a) $500 \mathrm{~nm}$ scale, (b) $200 \mathrm{~nm}$ scale and (c) $100 \mathrm{~nm}$ scale.

\section{FTIR analysis}

FTIR measurements were carried out to identify the major functional groups on the surface of the plant extract and their possible involvement in the capping and stabilization of the AgNPs. In Figure 5, FTIR spectra of AgNPs show a strong absorption band between $1500-1700 \mathrm{~cm}^{-1}$ and it is attributed to the binding of the extract's components including proteins and other organic molecules to the surface of the nanoparticles. The signals present at the $2000 \mathrm{~cm}^{-1}$ is also indicative of the attachment of $\mathrm{NHC}=\mathrm{O}$ to metal ions. Other peaks of interest include 2922 (secondary amine), 1383 (C-N stretching vibration of aromatic amine), 1138 and $821 \mathrm{~cm}^{-1}$ for AgNPs in aqueous C. occidentalis leaf extracts. The presence of peaks between 3100 and $3700 \mathrm{~cm}^{-1}$ could be due to the $\mathrm{O}-\mathrm{H}$ group in polyphenols or proteins or polysaccharide $[15,16]$. The phytochemical analysis of $C$. occidentalis has indicated the presence of phenolic compounds such as apigenin, emodin, aloe-emodin and rhein which are responsible for the formation or synthesis of metal nanoparticles. The presence of amide and carboxylic acid bonds in both samples confirmed the presence of protein polymers in the leaf extract which could be involved in the reduction of $\mathrm{Ag}^{+}$to $\mathrm{Ag}^{0}$ [12]. Biomolecules are known to interact with metal salts via these functional groups and aid their reduction to NPs [17]. These components not only aid in the capping and stabilizing of the nanoparticles, but also provide accessory components that give the nanoparticles their range of abilities, and in some cases amplifies them.

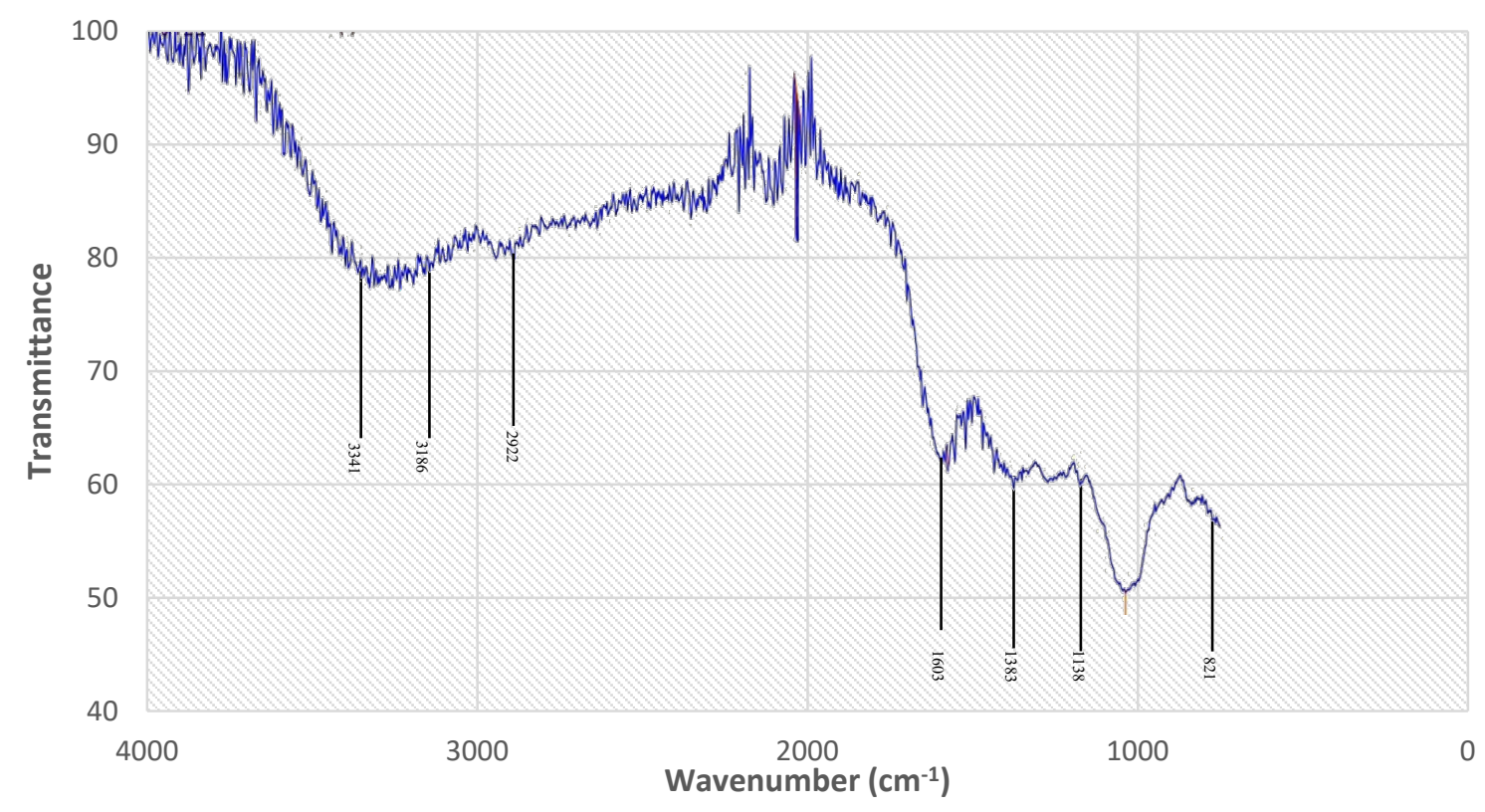

Figure 5 FTIR spectra of synthesized AgNPs. 


\section{Biocatalytic reduction of nitro organic compounds}

Reduction reactions for 4-nitrophenol (4-NP) and 4-nitroalanine (4-NA) using aqueous $\mathrm{NaBH}_{4}$ is thermodynamically favorable. However, the presence of the kinetic barrier due to the large potential difference between donor and acceptor molecules decreases the feasibility of this reaction. AgNPs overcome the kinetic barrier by facilitating electron relay from the donor $\mathrm{BH}_{4}$ - to acceptor 4-NP/4-NA. In the presence of AgNPs as a catalyst, the yellow color of the 4-NP solution gradually became clear and finally disappeared. In Figure 6, the UV-Vis absorption spectra revealed that the addition of AgNPs initiated the lowering of peak intensity at $400 \mathrm{~nm}$ and the presence of a new absorption peak at $280 \mathrm{~nm}$ indicating the formation of 4-aminophenol. The complete disappearance of the $400 \mathrm{~nm}$ peak was observed within $30 \mathrm{~min}$.

The reduction reaction of 4-NA can be monitored easily by UV-Vis spectroscopy by measuring the decrease in the absorbance intensity at $380 \mathrm{~nm}$. The reduction reaction of 4-NA by $\mathrm{NaBH}_{4}$ did not take place in the absence of the AgNPs as shown in Figure 7 but after the addition of the AgNPs, immediate reduction took place. This reduction was also observed visually by the steady disappearance of the yellow color. The reduction reaction was monitored every 5 min using UV-Vis spectroscopy. Figure 8 presents a probable mechanism for the catalytic reduction of 4-NP and 4-NA. In this catalytic reduction, $\mathrm{NaBH}_{4}$ acts as an electron donor and hydrogen supplier, the AgNPs are an electron transfer mediator that collects electron from $\mathrm{BH}_{4}{ }^{-}$ion to 4-NP and 4-NA [18]. The simultaneous adsorption of hydrogen species from $\mathrm{BH}_{4}^{-}, 4-\mathrm{NP}$ and 4-NA on the surface of AgNPs takes place and diffusion between the adsorbed species leads to desorption of the product [19]. The complete reduction of 4-NA results in the formation of 1,4 diaminobenzene after $30 \mathrm{~min}$.

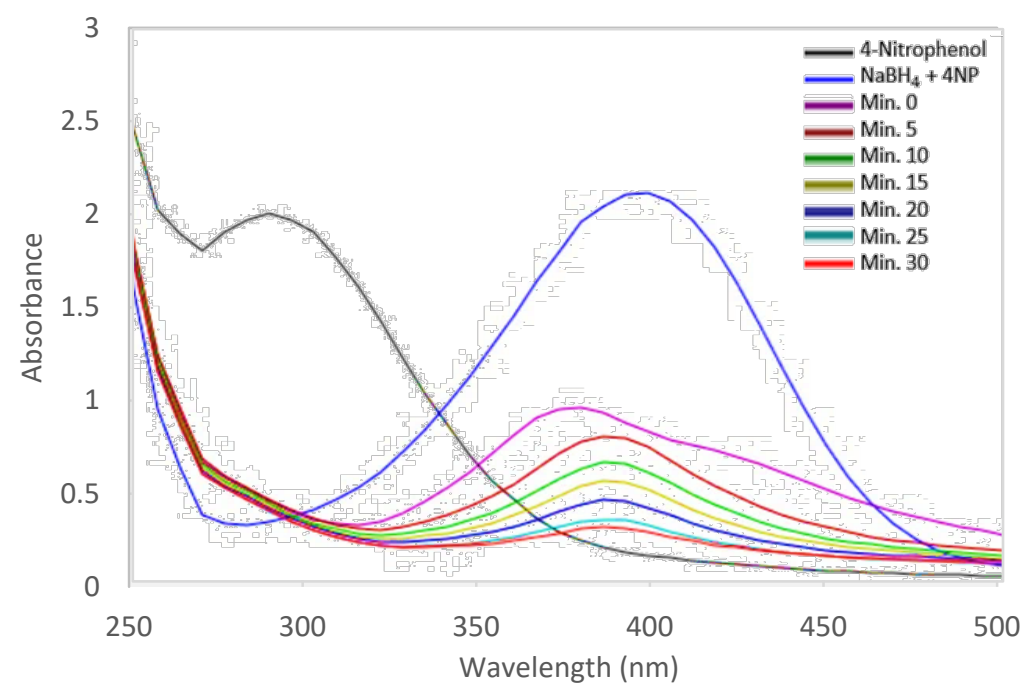

Figure $6 \mathrm{UV}$-Vis spectrum of the reduction of 4-nitrophenol (4-NP) by C. occidentalis/AgNPs conjugates. 


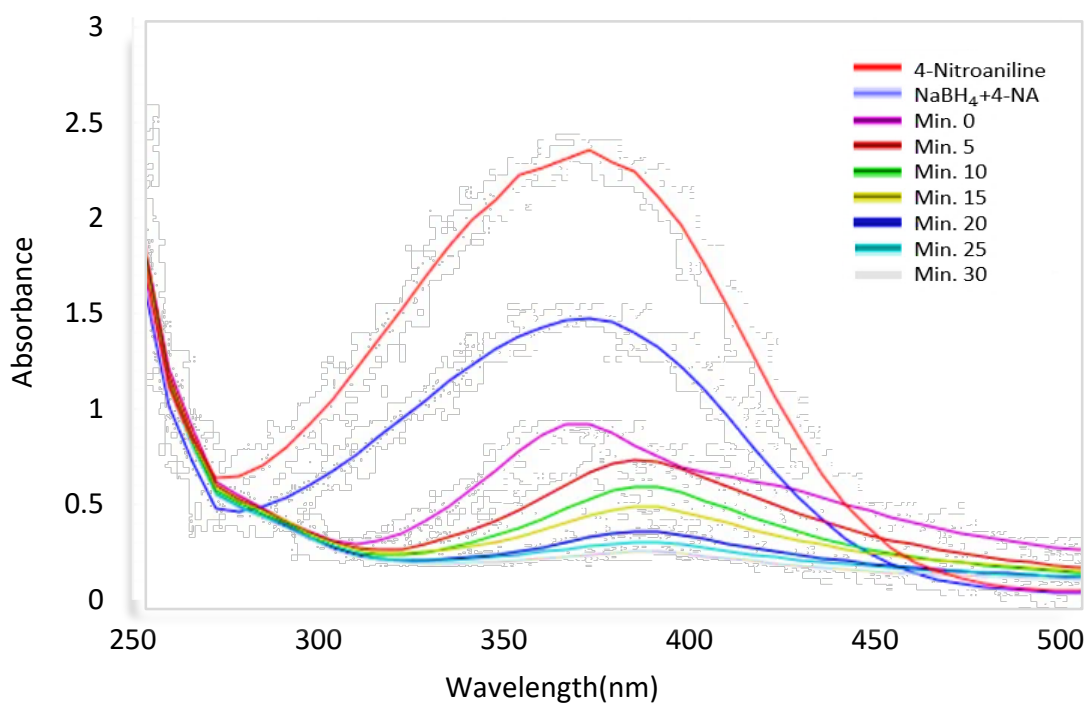

Figure 7 UV-Vis spectrum of the reduction of 4-nitroalanine (4-NA) by C. occidentalis/AgNPs conjugates.

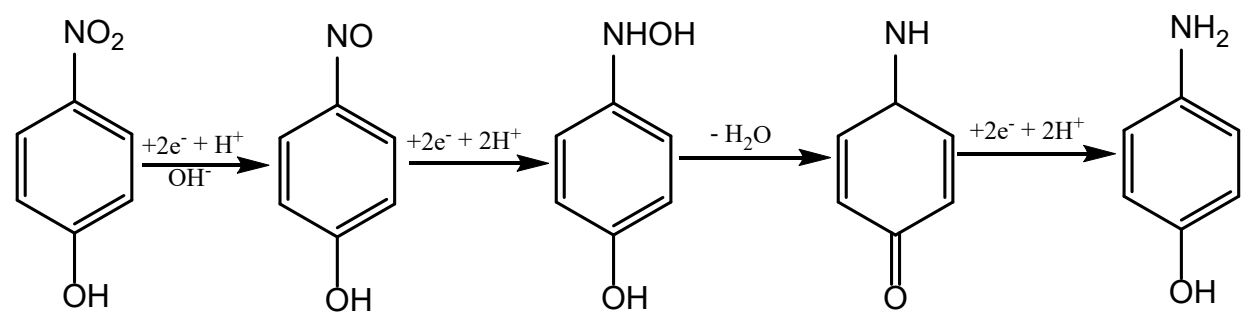

4-Nitrophenol (4-NP)

A<smiles>C1CC2CCC1C2</smiles>

B

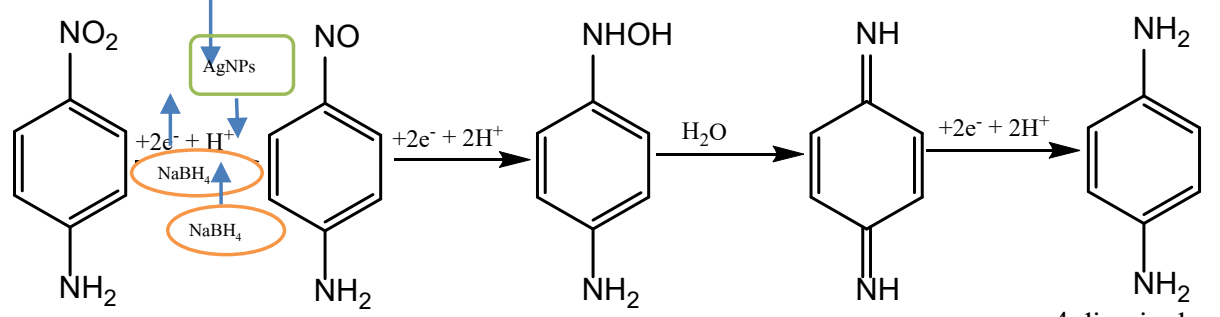

4-Nitroalanine (4-NA)

1, 4 diaminobenzene

Figure 8 Probable mechanism for the catalytic reduction of 4-NP (A) and 4-NA by $\mathrm{NaBH}_{4}$ using AgNPs as a catalyst.

\section{Antibacterial, antioxidant and cytotoxicity assays}

AgNPs are well known for their antimicrobial activity, which is said to be attributed to the electrostatic interaction that occurs between the negatively charged cell membrane of microorganism and the positively charged AgNPs [20]. The accumulation of AgNPs on the cell membrane is believed to alter the membrane causing it to lose permeability which leads to cell death [21]. However, due to chemical agents used when preparing these AgNPs, which affect their biocompatibility and pose environmental problems, biosynthesized AgNPs have been the subject of research recently.

Dimethyl sulfoxide (DMSO) was used as a negative control and proved to be a safe solvent, which offered no interference with the results obtained in the assay. The diameter of inhibition zones $(\mathrm{mm})$ 
around each well with AgNPs solution is presented in Table 1. Synthesized AgNPs exhibited good antimicrobial activity against both gram-positive and gram-negative bacteria, with varying degrees of inhibition (Table 1). Bacillus cereus had a slightly larger zone of inhibition $(5-10 \mathrm{~mm})$. Overall, these findings revealed that good antibacterial activity was achieved at higher doses of the $C$. occidentalis mediated AgNPs against all tested bacterial strains, suggesting that $C$. occidentalis mediated AgNPs were good antimicrobial agents. The efficacy of the AgNPs at this stage is not comparable to the ciprofloxacin's inhibiting ability however, due to differing concentrations, therefore until the concentration of the AgNPs is increased further, it may be simply noted as capable of inhibiting bacterial activity.

Table 1 Antibacterial activity of AgNPs of C. occidentalis.

\begin{tabular}{lccccc}
\hline \multirow{2}{*}{ Bacteria } & \multicolumn{4}{c}{ Zone of inhibition (mm) } \\
\cline { 2 - 5 } & $\begin{array}{c}\text { AgNP } \\
\mathbf{1 0 0 0} \boldsymbol{\mu g} / \mathbf{m l}\end{array}$ & $\begin{array}{c}\mathbf{A g N P} \\
\mathbf{5 0 0} \mathbf{\mu g} / \mathbf{m l}\end{array}$ & $\begin{array}{c}\mathbf{A g N P} \\
\mathbf{2 5 0} \boldsymbol{\mu g} / \mathbf{m l}\end{array}$ & $\begin{array}{c}\text { Ciprofloxacin } \\
\text { (+ control) }\end{array}$ & $\begin{array}{c}\text { DMSO } \\
\text { (- control) }\end{array}$ \\
\hline $\boldsymbol{E}$. coli & $8 \pm 0.1$ & $6 \pm 0.1$ & $6 \pm 0.1$ & $25 \pm 0.2$ & 0 \\
K. pneumoniae & $8 \pm 0.2$ & $5 \pm 0.2$ & $5 \pm 0.1$ & $22 \pm 0.3$ & 0 \\
$\boldsymbol{P}$. aeruginosa & $9 \pm 0.2$ & $6 \pm 0.1$ & $6 \pm 0.1$ & $25 \pm 0.5$ & 0 \\
S. aureus & $9 \pm 0.2$ & $6 \pm 0.2$ & $5 \pm 0.1$ & $15 \pm 0.1$ & 0 \\
B. cereus & $10 \pm 0.1$ & $7 \pm 0.1$ & $6 \pm 0.1$ & $22 \pm 0.5$ & 0 \\
E. faecalis & $7 \pm 0.1$ & $6 \pm 0.3$ & $5 \pm 0.2$ & $20 \pm 0.1$ & 0 \\
\hline
\end{tabular}

Nuño-Martínez (2019) stated that the electric charges on the surface of the bacteria have a drastic effect with its reaction to the charges of the nanoparticles, this point was proven by an experiment conducted by Abbaszadegan et al. (2015) who determined the effects of nanoparticles with different surface charges had different effects on bacteria. The results revealed that AgNPs with a positive surface charge was the most effective against all types of bacteria (gram-negative and gram-positive), however, AgNPs with a negative surface charge is the least effective against bacteria [23]. AgNPs in general has many mechanisms for causing cell death. AgNPs can cause cell death by being absorbed into the cells, once in the cell, it induces DNA damage and subsequent cell death [24]. According to Brunner et al. (2006), the toxicity of AgNPs may be due to the direct interaction with the nanoparticles and the biological macromolecules or the release of metal ions from the nanoparticle.

With the direct interaction, the AgNPs anchor themselves to the bacterial cell wall. This subsequently causes the cell wall to break apart and the nanoparticle can infiltrate the cell. Due to the membrane break, contents of the cell tend to leak out of the cell membrane, and this can cause cell death [26]. When the nanoparticles enter the cell, they may interact with cellular components such as proteins, lipids and DNA, this interaction leads to dysfunction and death of the cell. An example is an interaction between the ribosomes and the nanoparticles, the ribosomes become denatured and this, in turn, inhibits translation as well as protein synthesis. Another antibacterial mechanism involves the production of high levels of reactive oxygen species (ROS), such as hydroxyl radical, hydrogen peroxide, hypochlorous acid and superoxide anion. This ROS generated in excess in the cell leads to inhibition of respiration and inhibition of cell growth [27].

The toxicity of the AgNPs was minimal. AgNPs showed only $3 \%$ toxicity towards Artemia nauplii after $20 \mathrm{~h}$ (Table 2). Test samples with a cytotoxic effect lower than $50 \%$ are considered safe. The low toxicity of nanoparticles indicates that although they are toxic to bacterial cells, they will be safe for complex life forms such as brine shrimp and humans. Thus, these nanoparticles can be used in medicinal products, without the concern of damage to the human body, essentially these nanoparticles can be used as drug vectors, delivering active components with far more accuracy without any harmful effects [28]. This also indicates that these nanoparticles will pose no inherent threat to aquatic life in the marine environment [29]. The plant extract, ethanol and silver nitrate were also run to determine if the components have any toxicity individually; these also showed zero toxicity. Potassium Dichromate wax used as the positive control and exhibited a $100 \%$ kill rate. 
Table 2 The \% mortality of brine shrimp (Artemia nauplii) treated at various concentrations of AgNPs.

\begin{tabular}{lllllll}
\hline Concentration (ug/ml) & Hour 1 & Hour 2 & Hour 3 & Hour 4 & Hour 20 & Hour 24 \\
\hline Silver Nanoparticles & & & & & & \\
1000 & $0 \%$ & $0 \%$ & $0 \%$ & $0 \%$ & $3 \%$ & $3 \%$ \\
500 & $0 \%$ & $0 \%$ & $0 \%$ & $0 \%$ & $0 \%$ & $0 \%$ \\
250 & $0 \%$ & $0 \%$ & $0 \%$ & $0 \%$ & $0 \%$ & $0 \%$ \\
Potassium Dichromate & & & & & & \\
1000 & $67.67 \%$ & $100 \%$ & $100 \%$ & $100 \%$ & $100 \%$ & $100 \%$ \\
500 & $46,67 \%$ & $63,33 \%$ & $83,33 \%$ & $100 \%$ & $100 \%$ & $100 \%$ \\
250 & $30 \%$ & $50 \%$ & $66,67 \%$ & $80 \%$ & $100 \%$ & $100 \%$ \\
\hline
\end{tabular}

DPPH is a stable free radical scavenger and shows a characteristic absorption at a wavelength between 510-530 nm. Thereduction of DPPH by antioxidants to 1,1- diphenyl-2-picryl hydrazine results in a colour change from violet to yellow [30], which was observed during the analysis using AgNPs. The free radical scavenging activity of AgNPs at all concentrations (100, 250, 550 and $1000 \mu \mathrm{g} / \mathrm{mL}) \mathrm{was}$ found to be higher than that of the plant extract (Figure 9). This suggests that these nanoparticles can act as antioxidants and can protect oxidizable molecules by inhibiting or slowing down the autoxidation of these molecules. This is achieved by decreasing the initiation events or by trapping the chain carrying radicals. These nano antioxidants are exceptional since they can be designed specially to have extended stability compared to other antioxidants, and this avoids rapid metabolic clearance, and single out specific sites [31].

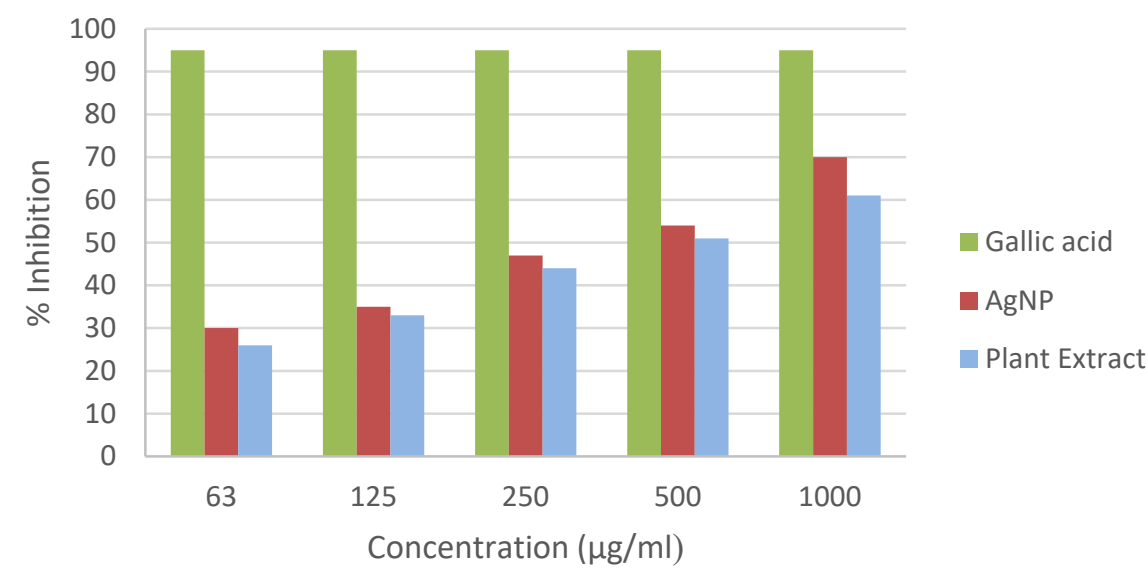

Figure 9 Antioxidant property of AgNPs using leaf extracts of C. occidentalis.

\section{Conclusions}

AgNPs were successfully synthesized using plant extract of $C$. occidentalis. The metal nanoparticles were characterized by UV-Vis, SEM-EDX, TEM and FTIR measurements. The formation of AgNPs was observed by a visible color change from yellow to brown and was confirmed by UVVisible spectroscopy. These AgNPs were very stable and showed antibacterial activity against a range of tested gram-positive and gram-negative bacteria at low doses, thus proving to be good antimicrobial agents. The AgNPs showed higher scavenging activity than leaf extracts of C. occidentalis. The AgNPs have increased catalytic ability for the reduction of 4-NP and 4-NA after a 30 min time interval. This research augments the application of green synthesized nanoparticles over chemically synthesized nanoparticles for the bioremediation of nitro organic waste. 


\section{Acknowledgements}

The National Research Foundation of South Africa and the Durban University of Technology are acknowledged for financial and infrastructural support.

\section{References}

[1] I Khan, K Saeed and I Khan. Nanoparticles: Properties, applications and toxicities. Arab J. Chem. 2017; 12, 908-31.

[2] JS Duhan, R Kumar, N Kumar, P Kaur, K Nehra and S Duhan. Nanotechnology: The new perspective in precision agriculture. Biotechnol. Rep. 2017; 15, 11-23.

[3] MZ Khan, FK Tareq, M Hossain and M Roki. Green synthesis and characterization of silver nanoparticles using Coriandrum sativum leaf extract. J. Eng. Sci. Technol. 2018; 13, 158-66.

[4] M Gondwal and G Joshi. Synthesis and catalytic and biological activities of silver and copper nanoparticles using Cassia occidentalis. Int. J. Biomater. 2018; 2018, 6735426.

[5] MA Ibrahim, AB Aliyu, AB Sallau, M Bashir, I Yunusa and TS Umar. Senna occidentalis leaf extract possesses antitrypanosomal activity and ameliorates the trypanosome-induced anaemia and organ damage. Pharmacogn. Res. 2010; 2, 175-80.

[6] MGB Silva, TP Aragão, CFB Vasconcelos, PA Ferreira, BA Andrade, IMA Costa, JH Costa-Silva, AG Wanderley and SSL Lafayette. Acute and subacute toxicity of Cassia occidentalis L. stem and leaf in wistar rats. J. Ethnopharmacol. 2011; 136, 341-46.

[7] S Vijayalakshmi, J Ranjitha, D Rajeswari and M Bhagiyalakshmi. Pharmacological profile of Cassia occidentalis L.: A review. Int. J. Pharm. Pharm. Sci. 2013; 5, 29-33.

[8] S Pal, P Kumar, E Ramakrishna, S Kumar, K Porwal, B Kumar, KR Arya, R Maurya and N Chattopadhyay. Extract and fraction of Cassia occidentalis L. (a synonym of Senna occidentalis) have an osteogenic effect and prevent glucocorticoid-induced osteopenia. J. Ethnopharmacol. 2019; 235, 8-18.

[9] XF Zhang, ZG Liu, W Shen and S Gurunathan. Silver nanoparticles: Synthesis, characterisation, properties, applications and therapeutic approaches. Int. J. Mol. Sci. 2016; 17, 1534.

[10] N Pradhan, A Pal and T Pal. Silver nanoparticle catalyzed reduction of aromatic nitro compounds, Colloids Surf. Physicochem. Eng. Asp. 2002; 196, 247-57.

[11] SS Shankar, A Ahmad and M Sastry. Geranium leaf assisted biosynthesis of silver nanoparticles. Biotechnol. Prog. 2003; 19, 1627-31.

[12] J Ashay. Rapid green synthesis of silver nanoparticles (AgNPs) using (Prunus persica) plant's extract: Exploring its antimicrobial and catalytic activities. Int. J. Nanomed. Nanotech. 2017; 8, 1000452 .

[13] SH Lee and BH Jun. Silver nanoparticles: Synthesis and application for nanomedicine. Int. J. Mol. Sci. 2019; 20, 865.

[14] P Banerjee, M Satapathy, A Mukhopahayay and P Das. Leaf extract mediated green synthesis of silver nanoparticles from widely available Indian plants: Synthesis, characterization, antimicrobial property and toxicity analysis. Bioresour. Bioprocess. 2014; $1,3$.

[15] JY Song, HK Jang and BS Kim. Biological synthesis of gold nanoparticles using Magnolia kobus and Diopyros kaki leaf extracts. Process Biochem. 2009; 44, 1133-8.

[16] H Susanto, Y Feng and M Ulbricht. Fouling behaviour of aqueous solutions of polyphenolic compounds during ultrafiltration. J. Food Eng. 2009; 91, 333-40.

[17] RA Hamouda, MH Hussein, RA Abo-Elmagd and SS Bazawir. Synthesis and biological characterization of silver nanoparticles derived from the cyanobacterium Oscillatoria limnetica. Sci. Rep. 2019; 9, 13071.

[18] A Gangula, R Podila, M Ramakrishna, L Karanam, C Janardhana and AM Rao. Catalytic reduction of 4-nitrophenol using biogenic gold and silver nanoparticles derived from Breynia rhamnoides. Langmuir 2011; 27, 15268-74.

[19] S Wunder, Y Lu, M Albrecht and M Ballauff. Catalytic activity of faceted gold nanoparticles studied by a model reaction: Evidence for substrate-induced surface restructuring. ACS Catal. 2011; 1, 908-16.

[20] YN Slavin, J Asnis, UO Häfeli and H Bach. Metal nanoparticles: Understanding the mechanisms behind the antibacterial activity. J. Nanobiotech. 2017; 15, 65.

[21] C Ramamurthy, M Padma, R Mareeswaran, A Suyavaran, MS Kumar, K Premkumar and C Thirunavukkarasu. The extra cellular synthesis of gold and silver nanoparticles and their free radical scavenging and antibacterial properties. Colloids Surf. B Biointerfaces 2013; 102, 808-15. 
[22] N Niño-Martínez, MF Salas Orozco, GA Martínez-Castañón, F Torres Méndez and F Ruiz. Molecular mechanisms of bacterial resistance to metal and metal oxide nanoparticles. Int. J. Mol. Sci. 2019; 20, 2808.

[23] A Abbaszadegan, Y Ghahramani, A Gholami, B Hemmateenejad, S Dorostkar, M Nabavizadeh and $\mathrm{H}$ Sharghi. The effect of charge at the surface of silver nanoparticles on antimicrobial activity against gram-positive and gram-negative bacteria: A preliminary study. J. Nanomat. 2015; 2015, 720654.

[24] Y Qing, L Cheng, R Li, G Liu, Y Zhang, X Tang, J Wang, H Liu and Y Qin. Potential antibacterial mechanism of silver nanoparticles and the optimization of orthopaedic implants by advanced modification technologies. Int. J. Nanomed. 2018; 13, 3311-27.

[25] TJ Brunner, P Wick, P Manser, P Spohn, RN Grass, LK Limbach, A Bruinink and WJ Stark. In vitro cytotoxicity of oxide nanoparticles: Comparison to asbestos, silica, and the effect of particle solubility. Environ. Sci. Technol. 2006; 40, 4374-81.

[26] SK Kailasa, TJ Park, JV Rohit and JR Koduru. Antimicrobial activity of silver nanoparticles. Nanopart. Pharmacother. 2019, 461-84.

[27] JK Patra and KH Baek. Green nanobiotechnology: Factors affecting synthesis and characterization techniques. J. Nanomat. 2014; 2014, 417305.

[28] C Arulvasu, SM Jennifer, D Prabhu and D Chandhirasekar. Toxicity effect of silver nanoparticles in brine shrimp Artemia. Sci. World J. 2014; 2014, 256919.

[29] HY Lin and CC Chou. Antioxidative activities of water-soluble disaccharide chitosan derivatives. Food Res. Int. 2014; 37, 883-9.

[30] L Valgimigli, A Baschieri and R Amorati. Antioxidant activity of nanomaterials. J Mater. Chem. B 2018; 6, 2036-51.

[31] N Pradhan, A Pal and T Pal. Silver nanoparticle catalyzed reduction of aromatic nitro compounds. Colloids Surf. Physicochem. Eng. Asp. 2002; 196, 247-57.

[32] S Shiravand and F Azarbani. Photosynthesis, characterization, antibacterial and cytotoxic effects of copper nanoparticles. Green Chem. Lett. Rev. 2017; 10, 241-249.

[33] MR Kalbassi, SA Johari, M Soltani and I Yu. Particle size and agglomeration affect the toxicity levels of silver nanoparticle types in the aquatic environment. Ecopersia 2013; 1, 273-90. 